\title{
Grouping of RFID Tags via Strongly Selective Families
}

\author{
Yi-Sheng Su, Jiun-Ren Lin, and Ozan K. Tonguz
}

\begin{abstract}
This letter proposes a novel scheme for grouping of radio-frequency identification (RFID) tags, based on strongly selective families (SSFs). Grouping of RFID tags allows verifying the integrity of groups of objects without external systems such as databases or verifiers, and can be extended to identify missing objects. The existing scheme is based on Gallager's low-density parity-check (LDPC) codes and, as such, it cannot easily achieve designated decoding guarantees due to its pseudo-random nature. Motivated by the strongly selective property of SSFs, this study proposes grouping of RFID tags via SSFs, such that designated decoding guarantees are more easily achieved. Simulation and theoretical results are provided, demonstrating that the proposed scheme can greatly improve the performance of the existing one.
\end{abstract}

Index Terms-Grouping of radio-frequency identification (RFID) tags, integrity, missing-object identification, strongly selective families (SSFs).

\section{INTRODUCTION}

Radio-frequency identification (RFID) [1] is an automatic identification technology. At its most basic, an RFID system consists of tags and interrogators. By wirelessly interrogating tags attached to physical objects, interrogators can obtain data stored on tags, such as identity of individual objects.

RFID has various applications. In applications such as incoming shipment inspection, verifying the integrity of a group of RFID tags or identifying missing RFID tags is beneficial to locate missing RFID tagged objects. Such functionalities are usually realized either by looking up the list of grouped objects or from the perspective of security. In either way, interrogators need access to external systems such as databases or verifiers. To eliminate the requirement for external systems, Sato et al. [2] recently proposed a new group verification scheme, also known as grouping ${ }^{1}$ of RFID tags, or grouping for short, which relies on group generation matrices to determine the number of missing RFID tags. They also extended grouping to identify the unique identifiers (IDs) of missing RFID tags [3]. In general, it is desirable to have either guaranteed determination of the number of missing RFID tags or guaranteed identification of missing RFID tags. For these purposes, group generation matrices used in grouping and its extended counterpart need to have certain properties. However, the existing scheme [2], [3] did not address such an issue and just used the paritycheck matrices of Gallager's low-density parity-check (LDPC) codes as group generation matrices. Due to the pseudo-random nature of Gallager's LDPC codes, the existing scheme cannot easily achieve designated decoding guarantees.

Motivated by the strongly selective property of strongly selective families (SSFs), this study proposes a novel scheme based on SSFs to construct group generation matrices, such

Y.-S. Su is with the Department of Computer Science and Information Engineering, Chang Jung Christian University, Tainan, Taiwan, ROC.

J.-R. Lin and O. K. Tonguz are with the Department of Electrical and Computer Engineering, Carnegie Mellon University, Pittsburgh, PA 152133890 USA.

${ }^{1}$ To avoid confusion with coding techniques with algebraic groups, grouping is used instead of group coding [2], [3]. that designated decoding guarantees are more easily achieved. Simulation and theoretical results are provided, demonstrating that the proposed scheme can provide great improvements over the existing one.

\section{PREliminaries}

This section briefly introduces grouping and its extended counterpart, and it also reviews the existing scheme. For details, refer to [2], [3].

\section{A. Grouping}

Grouping employs the check sum technique to check the integrity of a group. The check sum of a group is calculated as the bitwise exclusive or (XOR) of all hashes of the unique IDs of the RFID tags belonging to the group, where a hash function is used to avoid zero check sums, and it serves as an ID of the group, i.e., group ID. In this context, the hashes of RFID tags and group IDs are viewed as binary row vectors.

1) Encoding: To determine the number of RFID tags missing from a target group, grouping divides the target group into overlapping subgroups and then calculates a group ID for each subgroup. The relation between the group IDs of all the subgroups and the hashes of all the RFID tags can be expressed as follows:

$$
\left[\begin{array}{c}
\mathbf{g}_{1} \\
\vdots \\
\mathbf{g}_{m}
\end{array}\right]=\mathbf{A} \cdot\left[\begin{array}{c}
\mathbf{h}_{1} \\
\vdots \\
\mathbf{h}_{n}
\end{array}\right]
$$

where $m$ denotes the number of subgroups, $\mathbf{g}_{i}, i \in[m] \triangleq$ $\{1, \ldots, m\}$, is the group ID of the $i^{\text {th }}$ subgroup, $n$ denotes the number of RFID tags, $\mathbf{h}_{j}, j \in[n]$, is the hash of the $j^{\text {th }}$ RFID tag, and $\mathbf{A}$ is an $m \times n\{0,1\}$-valued matrix, called the group generation matrix. The $(i, j)$ entry of $\mathbf{A}$, denoted by $\mathbf{A}_{i, j}$, is equal to one if the $i^{\text {th }}$ subgroup contains the $j^{\text {th }}$ RFID tag and is equal to zero otherwise. To eliminate the requirement for the whole A upon decoding, grouping writes each group ID only to the memory of those RFID tags belonging to the subgroup.

2) Decoding: By wirelessly interrogating RFID tags without errors, an interrogator can obtain the unique IDs of RFID tags and their involved subgroups' group IDs. Then it uses the hash function to verify the integrity of a subgroup. According to the verification results, (1) can be rearranged to the following form:

$$
\left[\begin{array}{c}
\mathrm{g}^{\mathbf{u}} \\
\hline \mathrm{g}^{\mathbf{c}} \\
\hline \mathrm{g}^{\mathbf{e}}
\end{array}\right]=\left[\begin{array}{c|c}
\mathbf{0} & \mathbf{A}^{\mathrm{um}} \\
\hline \mathbf{A}^{\mathbf{c r}} & \mathbf{0} \\
\hline \mathbf{A}^{\mathrm{er}} & \mathbf{A}^{\mathbf{e m}}
\end{array}\right] \cdot\left[\begin{array}{l}
\mathbf{h}^{\mathbf{r}} \\
\hline \mathbf{h}^{\mathbf{m}}
\end{array}\right] \text {. }
$$

In (2), $\mathbf{g}^{\mathbf{u}}$ is the array of group IDs of the subgroups that are unknown because all the RFID tags belonging to these subgroups are missing, while $\mathbf{g}^{\mathbf{c}}$ and $\mathbf{g}^{\mathbf{e}}$ are those that preserve and lose their integrity, respectively. Likewise, $\mathbf{h}^{\mathbf{r}}$ and $\mathbf{h}^{\mathbf{m}}$ are the arrays of hashes of the RFID tags read by the interrogator and missing from the target group, respectively. According 
to these arrays, $\mathbf{A}$ can be rearranged and separated into six submatrices $\mathbf{0}, \mathbf{A}^{\mathbf{c r}}, \mathbf{A}^{\mathbf{e r}}, \mathbf{A}^{\mathbf{u m}}, \mathbf{0}$ and $\mathbf{A}^{\mathbf{e m}}$, where $\mathbf{0}$ is the zero matrix. Because the information of $\mathbf{A}$ is distributed across RFID tags, $\mathbf{A}^{\mathbf{u m}}$ and $\mathbf{A}^{\mathbf{e m}}$ are not available upon decoding. The following result, however, can be obtained from (2):

$$
\mathbf{A}^{\mathbf{e m}} \mathbf{h}^{\mathbf{m}}=\mathbf{g}^{\mathbf{e}} \oplus \mathbf{A}^{\mathbf{e r}} \mathbf{h}^{\mathbf{r}}
$$

where $\oplus$ denotes the bitwise XOR operator. Assuming $\mathbf{h}^{\mathbf{m}}$ is an array of the mutually independent hashes of missing RFID tags' unique IDs, the number of missing RFID tags is equal to the rank of $\mathbf{h}^{\mathbf{m}}$. If $\mathbf{A}^{\mathbf{e m}}$ can preserve the rank of $\mathbf{h}^{\mathbf{m}}$, the number of missing RFID tags can be accurately determined by calculating the rank of $\mathbf{A}^{\mathbf{e m}} \mathbf{h}^{\mathbf{m}}$. Under this condition, because $\mathbf{g}^{\mathbf{e}}, \mathbf{A}^{\mathbf{e r}}$ and $\mathbf{h}^{\mathbf{r}}$ are known, (3) implies that calculating the rank of $\mathbf{g}^{\mathbf{e}} \oplus \mathbf{A}^{\mathbf{e r}} \mathbf{h}^{\mathbf{r}}$ determines the accurate number of missing RFID tags, regardless of the unavailability of $\mathbf{A}^{\mathbf{e m}}$ and $\mathbf{h}^{\mathbf{m}}$.

\section{B. Extended Grouping}

As seen in (3), the unknown submatrix $\mathbf{A}^{\mathbf{e m}}$ is the main difficulty for grouping to identify missing RFID tags. To overcome the problem, extended grouping writes additional information to the memory of RFID tags upon encoding.

1) Encoding: In addition to group IDs, extended grouping assigns a short ID to each RFID tag belonging to a target group. The short ID is required to be unique within the target group, and because the target group size is generally much less than the maximum number of RFID tags, a portion of factoryprogrammed tag ID is enough to practically serve the purpose. Therefore, each RFID tag in a subgroup not only stores the group ID of this subgroup, but also the short IDs of the other RFID tags belonging to this subgroup. Additional difference from grouping is that the hash function for extended grouping should be the identity hash function, as the aim of extended grouping is to identify the unique IDs of missing RFID tags.

2) Decoding: An interrogator can retrieve $\mathbf{A}^{\mathbf{e m}}$ from the short IDs of missing RFID tags. As a result, (3) represents a set of linear equations with only $\mathbf{h}^{\mathbf{m}}$ unknown, and it defines a bipartite graph consisting of two sets of nodes: one of which represents the missing RFID tags, and the other the incomplete subgroups. In the bipartite graph, an edge connects a missing-RFID-tag node to an incomplete-subgroup node if the missing RFID tag belongs to the incomplete subgroup. While representing (3) as a bipartite graph, a simple attempt to solve (3) is by iterative message passing [3].

\section{Existing Scheme}

The existing scheme used parity-check matrices of Gallager's LDPC codes as group generation matrices, henceforth referred to as the LDPC-based scheme.

Definition 1 ([4]): An $(n, j, k)$ LDPC code is a code of block length $n$ with a parity-check matrix that has $j$ ones in each column, $k$ ones in each row, and zeros elsewhere.

Example 1: The following is an example of parity-check matrices of a $(9,3,3)$ Gallager's LDPC code [4]:

$$
\mathbf{A}=\left[\begin{array}{lllllllll}
1 & 1 & 1 & 0 & 0 & 0 & 0 & 0 & 0 \\
0 & 0 & 0 & 1 & 1 & 1 & 0 & 0 & 0 \\
0 & 0 & 0 & 0 & 0 & 0 & 1 & 1 & 1 \\
\hline 0 & 0 & 1 & 0 & 1 & 0 & 1 & 0 & 0 \\
1 & 0 & 0 & 0 & 0 & 0 & 0 & 1 & 1 \\
0 & 1 & 0 & 1 & 0 & 1 & 0 & 0 & 0 \\
\hline 1 & 0 & 1 & 0 & 0 & 0 & 1 & 0 & 0 \\
0 & 0 & 0 & 1 & 0 & 0 & 0 & 1 & 1 \\
0 & 1 & 0 & 0 & 1 & 1 & 0 & 0 & 0
\end{array}\right] .
$$

The matrix in (4) is divided into three submatrices, each containing a single one in each column. As a group generation matrix, this matrix can divide a group of $n=9$ RFID tags into $m=9$ overlapping subgroups such that each subgroup contains $k=3$ RFID tags and each RFID tag belongs to $j=3$ subgroups. Because the second and third submatrices are random column permutations of the first, the columns in (4) are not necessarily linearly independent, e.g., columns 8 and 9. From columns 8 and $9, \mathbf{A}^{\mathbf{e m}}=\left[\begin{array}{lll}1 & 1 & 1 \\ 1 & 1 & 1\end{array}\right]^{\top}$ with rank one, where $(\cdot)^{\top}$ is the transpose operator, under which neither the rank of $\mathbf{h}^{\mathbf{m}}$ is preserved nor iterative message passing can identify the two missing RFID tags.

\section{PROposed SCHEME}

This section first presents the background of SSFs [5], and then applies SSFs to the design of grouping of RFID tags. In what follows, let $[n]$ be the universe.

\section{A. Strongly Selective Families (SSFs)}

An SSF is a family of subsets of $[n]$ that selects any element out of any small enough subset of $[n]$.

Definition $2([5])$ : Let $r \leq n$. A family $\mathcal{F}$ of subsets of $[n]$ is said to be $(n, r)$-strongly-selective if, for any subset $X \subset[n]$ of size (at most) $r$ and for every element $x \in X$, there is a set $F$ in $\mathcal{F}$ such that $F$ selects $x$, i.e., $F \cap X=\{x\}$. Such a family is called an $(n, r)$-SSF.

Example 2: The following nine subsets of [9]:

$$
\begin{array}{lll}
\{1,2,3\}, & \{4,5,6\}, & \{7,8,9\}, \\
\{1,6,8\}, & \{2,4,9\}, & \{3,5,7\}, \\
\{1,5,9\}, & \{3,4,8\}, \quad\{2,6,7\},
\end{array}
$$

specify a $(9,3)$-SSF. To see this, consider any arbitrary subset of size at most three, e.g., $X=\{7,8,9\}$. Clearly, elements 7,8 and 9 are selected by subsets $\{2,6,7\},\{3,4,8\}$ and $\{1,5,9\}$, respectively.

\section{B. SSF-Based Scheme}

To reveal the motivation of grouping of RFID tags via SSFs, let us consider Example 2. Suppose that [9] represents the target group of RFID tags and that the subsets in Example 2 denote the subgroup structure. If $X$ is a set of missing RFID tags, one can see that the missing RFID tags can be identified easily by iterative message passing, as each of them is selected by a subset/subgroup in Example 2.

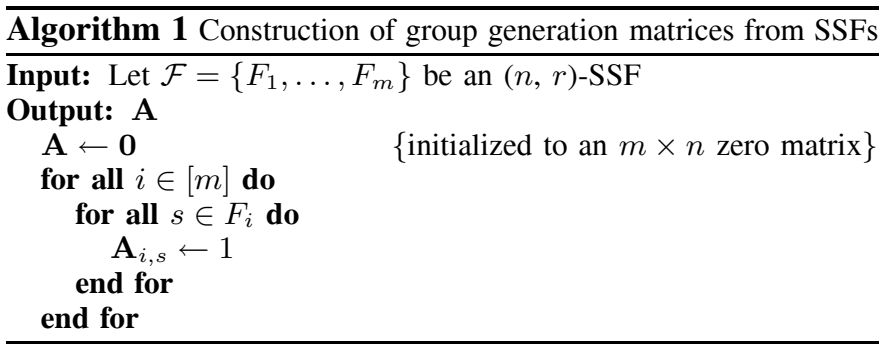

For grouping of RFID tags via SSFs, use Algorithm 1 to construct a group generation matrix $\mathbf{A}$ of size $m \times n$, which can divide $n$ RFID tags into $m$ overlapping subgroups. Such an approach is referred to as the SSF-based scheme and is demonstrated in the following example. 
Example 3: The group generation matrix constructed from the (9, 3)-SSF in Example 2 is shown as follows:

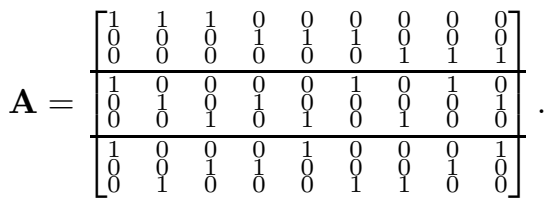

Similar to (4), the matrix in (5) consists of three submatrices, each containing a single one in each column. The first submatrix in (5) is also the same as that in (4). The second and third submatrices, however, are no longer randomly chosen column permutations of the first. From columns 8 and 9, $\mathbf{A}^{\mathrm{em}}=\left[\begin{array}{llll}1 & 1 & 0 & 0 \\ 1 & 0 & 1 & 1 \\ ]^{\top}\end{array}\right]^{\top}$ with rank two, under which the rank of $\mathbf{h}^{\mathbf{m}}$ is preserved and iterative message passing can identify the two missing RFID tags.

Remark 1: One can see that the bitwise OR of any set of at most two columns of (5) covers only the columns of the set, where a binary vector $\mathbf{x}$ is said to be covered in a binary vector $\mathbf{y}$ if and only if the bitwise OR of $\mathbf{x}$ and $\mathbf{y}$ is equal to $\mathbf{y}$. This is due to the strongly selective property of SSFs. Therefore, the columns of the matrix constructed from an SSF form a superimposed code [6]. In combinatorics, superimposed codes are known as cover-free families [7] and also closely connected to nonadaptive group testing [8].

One can see that every subset of three columns in (5) contains a $3 \times 3$ identity submatrix. This is due to the strongly selective property of SSFs. Then the following property holds.

Property 1: In A constructed from an $(n, r)$-SSF, every subset of $r$ columns contains an $r \times r$ identity submatrix.

By Property 1, the SSF-based scheme guarantees that any number of missing RFID tags not greater than $r$ is determined accurately and that any subset of up to $r$ missing RFID tags is identified. For comparison with the LDPC-based scheme, let $P_{I}(s)$ denote the probability that every subset of $s$ columns of a group generation matrix contains an $s \times s$ identity submatrix. Then the following are some results on $P_{I}(s)$.

Proposition 1: For A constructed from an $(n, r)-\mathrm{SSF}$, $P_{I}(s)=1$ if $1 \leq s \leq r$, while for $\mathbf{A}$ constructed from an $(n, j, k)$ Gallager's LDPC code, $P_{I}(s) \leq\left(1-\left(\frac{k-1}{n-1}\right)^{j}\right)^{\left(\begin{array}{c}s \\ 2\end{array}\right)}$ if $1 \leq s \leq \frac{n}{k}$.

Proof: The first part follows immediately from Property 1 . For the second part, one can see that any two arbitrary columns of the first submatrix of $\mathbf{A}$ are exactly the same with probability $\frac{k-1}{n-1}$. Moreover, in every subset of $s \leq \frac{n}{k}$ columns of $\mathbf{A}$, there are $\left(\begin{array}{l}s \\ 2\end{array}\right)$ pairs of columns, each of which contains a $2 \times 2$ identity submatrix with probability $1-\left(\frac{k-1}{n-1}\right)^{j}$ independently, according to the construction of Gallager's LDPC codes. Therefore, $P_{I}(s)$ can be bounded from above, as described above.

\section{Performance Evaluation}

This study conducts simulation to evaluate the error-rate performance of the proposed SSF-based scheme and compares it with that of the LDPC-based one. The error rate is the percentage of decoding failures, where a decoding failure means that, after finishing decoding, the number of missing RFID tags is not determined accurately or all the missing RFID tags cannot be identified. The simulation results presented below are mean values of $10^{7}$ simulation runs. In each simulation run, each unique ID is 96 bits in length and generated randomly, while the hashes of unique IDs for grouping are calculated using CRC-32. Missing RFID tags are selected randomly from the target group. For comparison with [2], [3], this study uses the parameters $(n, j, k)$ of LDPC codes to denote the structure of group generation matrices. Because aiming at minimizing the memory consumption of each RFID tag, this study considers two SSFs based on maximal-distance separable (MDS) $q$-ary codes [6]: $\left(q^{2}, 2\right)$ - and $\left(q^{2}, 3\right)$-SSFs, leading to group generation matrices with $\left(q^{2}, 2, q\right)$ and $\left(q^{2}, 3, q\right)$, respectively, where $q$ is a prime power. The SSF construction is detailed in the Appendix. For simplicity, the target group size is assumed to be equal to $n$. However, if the actual target group size is not fitted into $n$, select large enough parameters to construct a group generation matrix, and then remove some columns from the matrix.

Fig. 1 depicts the error rates of the LDPC- and SSF-based grouping schemes with different $(n, j, k)$ 's. Fig. 1 shows that the SSF-based scheme has lower error rates than the LDPCbased one. Moreover, Fig. 1 shows that a larger value of $k$ not only supports a larger target group of RFID tags, but also reduces error rates. Table I depicts the maximum number of missing RFID tags that can be determined within a specified tolerance of error rate. Table I shows that the maximum number of missing RFID tags that can be determined by the SSF-based scheme is higher than that provided by the LDPCbased one and that the decoding guarantee of the SSF-based scheme is greater than that determined by Property 1 .

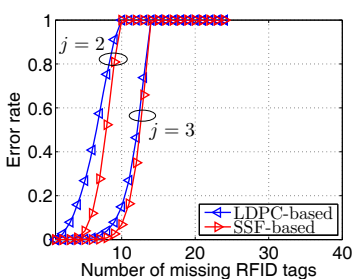

(a)

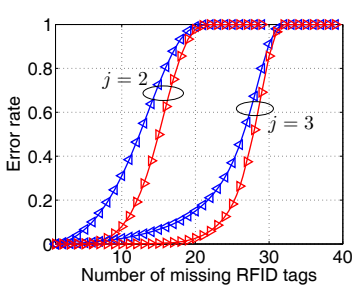

(c)

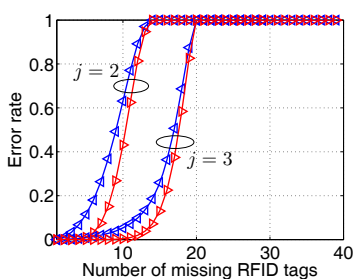

(b)

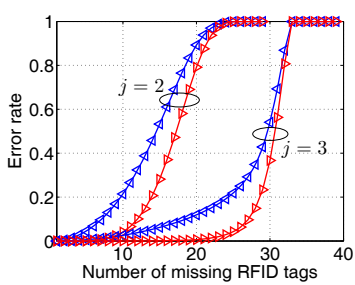

(d)
Fig. 1. Error rates of the LDPC- and SSF-based grouping schemes: (a) $(25, j, 5)$; (b) $(49, j, 7)$; (c) $(121, j, 11)$; (d) $(169, j, 13)$.

Fig. 2 depicts the error rates of different extended grouping schemes. Similar to grouping, Fig. 2 shows that the SSF-based scheme performs better than the LDPC-based one, especially when the number of missing RFID tags is not large. Table II depicts the maximum number of missing RFID tags that can be identified within a specified tolerance of error rate. Table II shows that the SSF-based scheme not only identifies more 
TABLE I

MAXIMUM NUMBER OF MISSING RFID TAGS THAT CAN BE DETERMINED WITHIN A SPECIFIED TOLERANCE OF ERROR RATE

\begin{tabular}{c|c|c|c|c|c}
\hline \multicolumn{2}{c|}{ Scheme } & \multicolumn{2}{c|}{ LDPC-based } & \multicolumn{2}{c}{ SSF-based } \\
\hline \multicolumn{2}{c|}{ Error rate } & $0 \%$ & $5 \%$ & $0 \%$ & $5 \%$ \\
\hline \hline \multirow{4}{*}{$(n, j, k)$} & $(25,2,5)$ & 1 & 2 & 3 & 5 \\
\cline { 2 - 6 } & $(49,2,7)$ & 1 & 3 & 3 & 6 \\
\cline { 2 - 6 } & $(121,2,11)$ & 1 & 4 & 3 & 9 \\
\cline { 2 - 6 } & $(169,2,13)$ & 1 & 5 & 3 & 10 \\
\hline \hline \multirow{4}{*}{$(n, j, k)$} & $(25,3,5)$ & 3 & 8 & 5 & 9 \\
\cline { 2 - 6 } & $(49,3,7)$ & 1 & 9 & 5 & 13 \\
\cline { 2 - 6 } & $(121,3,11)$ & 1 & 13 & 5 & 21 \\
\cline { 2 - 6 } & $(169,3,13)$ & 1 & 13 & 5 & 25 \\
\hline
\end{tabular}

missing RFID tags than the LDPC-based one, but also has stronger decoding guarantees compared to Property 1.

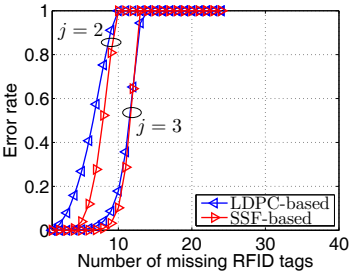

(a)

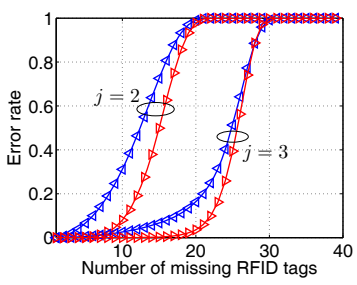

(c)

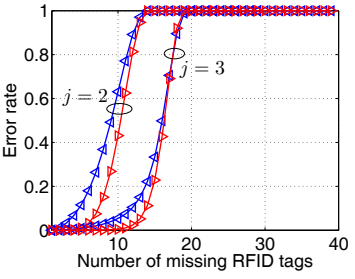

(b)

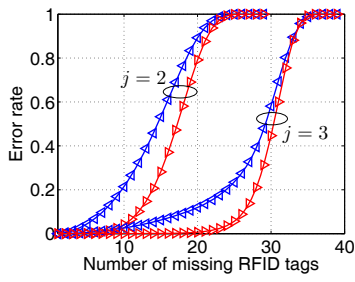

(d)
Fig. 2. Error rates of the LDPC- and SSF-based extended grouping schemes: (a) $(25, j, 5)$; (b) $(49, j, 7)$; (c) $(121, j, 11)$; (d) $(169, j, 13)$.

TABLE II

MAXIMUM NUMBER OF MISSING RFID TAGS THAT CAN BE IDENTIFIED WITHIN A SPECIFIED TOLERANCE OF ERROR RATE

\begin{tabular}{c|c|c|c|c|c}
\hline \multicolumn{2}{c|}{ Scheme } & \multicolumn{2}{c|}{ LDPC-based } & \multicolumn{2}{c}{ SSF-based } \\
\hline \multicolumn{2}{c|}{ Error rate } & $0 \%$ & $5 \%$ & $0 \%$ & $5 \%$ \\
\hline \hline \multirow{4}{*}{$(n, j, k)$} & $(25,2,5)$ & 1 & 2 & 3 & 5 \\
\cline { 2 - 6 } & $(49,2,7)$ & 1 & 3 & 3 & 6 \\
\cline { 2 - 6 } & $(121,2,11)$ & 1 & 4 & 3 & 9 \\
\cline { 2 - 6 } & $(169,2,13)$ & 1 & 5 & 3 & 10 \\
\hline \hline \multirow{4}{*}{$(n, j, k)$} & $(25,3,5)$ & 3 & 8 & 5 & 9 \\
\cline { 2 - 6 } & $(49,3,7)$ & 1 & 9 & 5 & 12 \\
\cline { 2 - 6 } & $(121,3,11)$ & 1 & 13 & 5 & 20 \\
\cline { 2 - 6 } & $(169,3,13)$ & 1 & 13 & 5 & 24 \\
\hline
\end{tabular}

This study also presents theoretical results about $P_{I}(s)$ of the LDPC- and SSF-based schemes, which are depicted in Fig. 3. As expected, Fig. 3 shows that, under the guarantee of decoding, the SSF-based scheme outperforms the LDPC-based one. Fig. 3 also shows that the gap between the two schemes vanishes as $j$ or $q$ increases. This is because, for the LDPCbased scheme, a large value of $j$ or $q$ increases the probability that each pair of columns has a $2 \times 2$ identity submatrix.

\section{CONClusion}

This letter has presented a strongly-selective-family-based scheme for dividing a target group of RFID tags into overlapping subgroups such that designated decoding guarantees are more easily achieved. Simulation and theoretical results

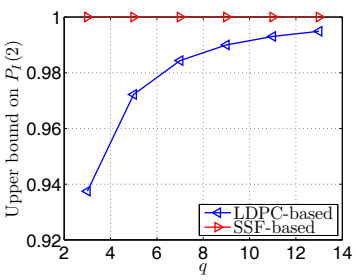

(a)

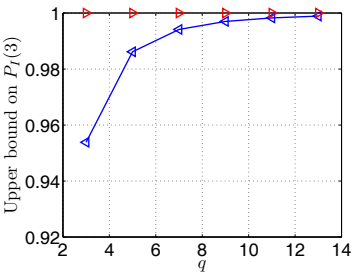

(b)
Fig. 3. Upper bound on $P_{I}(s)$ of the LDPC- and SSF-based schemes: (a) $\left(q^{2}, 2, q\right)$; (b) $\left(q^{2}, 3, q\right)$.

have showed that the proposed scheme can make great performance enhancements over that based on Gallager's lowdensity parity-check codes.

\section{APPENDIX SSF CONSTRUCTION}

The SSF construction is based on $[N, K]_{q}$ MDS codes over a finite field $\mathbb{F}_{q}$ with $q$ elements, where $N$ and $K$ are the block length and dimension of the code, respectively. Suppose $\mathbb{F}_{q}$ is represented by $[q]$ and $\mathcal{C}=\left\{\mathbf{c}_{1}, \ldots, \mathbf{c}_{q^{K}}\right\}$ is an $[N, K]_{q}$ MDS code over $\mathbb{F}_{q}$, where $\mathbf{c}_{i}=\left[c_{i, 1} \cdots c_{i, N}\right], i \in\left[q^{K}\right]$, is a codeword. The SSF $\mathcal{F}$ constructed from $\mathcal{C}$ consists of the sets of indices of codewords in $\mathcal{C}$ that have a certain element in a certain position. Let $F_{(s-1) q+t}=\left\{i \in\left[q^{K}\right] \mid c_{i, s}=t\right\}$ where $s \in[N]$ and $t \in[q]$. Then $\mathcal{F}=\left\{F_{(s-1) q+t} \mid s \in[N], t \in[q]\right\}$. The algorithm for the SSF construction is derived from the results in [6] and described in detail below.

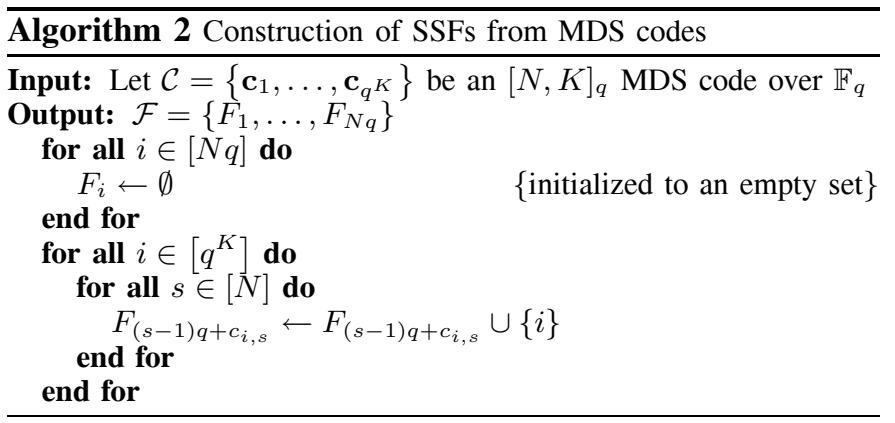

Among $[N, K]_{q}$ MDS codes with fixed $N$, the maximum decoding guarantee determined by Property 1 is achieved by those with $K=2$ [6]. Therefore, the SSFs used in Section IV are based on $[N, 2]_{q}$ MDS codes, which are further punctured to minimize the memory consumption of each RFID tag.

\section{REFERENCES}

[1] M. Bolic, D. Simplot-Ryl, and I. Stojmenovic, RFID Systems: Research Trends and Challenges, New York: Wiley, 2010.

[2] Y. Sato, J. Mitsugi, O. Nakamura, and J. Murai, "Theory and performance evaluation of group coding of RFID tags," IEEE Trans. Autom. Sci. Eng., vol. 9, pp. 458-466, July 2012.

[3] Y. Sato, Y. Igarashi, J. Mitsugi, O. Nakamura, and J. Murai, "Identification of missing objects with group coding of RF tags," in Proc. IEEE RFID, Apr. 2012, pp. 95-101.

[4] R. Gallager, "Low-density parity-check codes," IRE Trans. Inf. Theory, vol. 8, pp. 21-28, Jan. 1962.

[5] A. Clementi, A. Monti, and R. Silvestri, "Distributed broadcast in radio networks of unknown topology," Theoret. Comput. Sci., vol. 302, pp. 337-364, 2003.

[6] W. Kautz and R. Singleton, "Nonrandom binary superimposed codes," IEEE Trans. Inf. Theory, vol. 10, pp. 363-377, Oct. 1964.

[7] P. Erdös, P. Frankl, and Z. Füredi, "Families of finite sets in which no set is covered by the union of $r$ others," Israel J. Math., vol. 51, pp. 79-89, 1985.

[8] D.-Z. Du and F. K. Hwang, Combinatorial Group Testing and Its Applications, 2nd ed. Singapore: World Scientic, 2000. 